\section{Living with Lethal PIP3 Levels: Viability of Flies Lacking PTEN Restored by a PH Domain Mutation in Akt/PKB}

\author{
Hugo Stocker, ${ }^{1}$ Mirjana Andjelkovic, ${ }^{2 *}$ Sean Oldham, ${ }^{1}$ \\ Muriel Laffargue, ${ }^{3}$ Matthias P. Wymann, ${ }^{3}$ Brian A. Hemmings, ${ }^{2}$ \\ Ernst Hafen ${ }^{1} \dagger$
}

The phosphoinositide phosphatase PTEN is mutated in many human cancers. Although the role of PTEN has been studied extensively, the relative contributions of its numerous potential downstream effectors to deregulated growth and tumorigenesis remain uncertain. We provide genetic evidence in Drosophila melanogaster for the paramount importance of the protein kinase Akt [also called protein kinase $B(P K B)]$ in mediating the effects of increased phosphatidylinositol 3,4,5-trisphosphate (PIP3) concentrations that are caused by the loss of PTEN function. A mutation in the pleckstrin homology (PH) domain of Akt that reduces its affinity for PIP3 sufficed to rescue the lethality of flies devoid of PTEN activity. Thus, Akt appears to be the only critical target activated by increased PIP3 concentrations in Drosophila.

Mutations in the tumor suppressor gene PTEN (the phosphatase and tensin homolog on chromosome 10) are frequent in glioblastomas, endometrial carcinoma, melanomas, and prostate cancer (1). Furthermore, two dominant hamartoma syndromes, Cowden disease and Bannayan-Zonana syndrome, are linked to germ line mutations in PTEN (1). The PTEN protein carries a phosphatase domain resembling those of dual-specificity

'Zoologisches Institut der Universität Zürich, Winterthurerstrasse 190, CH-8057 Zürich, Switzerland. ${ }^{2}$ Friedrich Miescher Institute, Maulbeerstrasse 66, $\mathrm{CH}$ 4058 Basel, Switzerland. ${ }^{3}$ Université de Fribourg, Rue du Musée 5, CH-1700 Fribourg, Switzerland.

*Present address: Department of Vascular and Metabolic Diseases, F. Hoffmann-La Roche AG, CH-4070 Basel, Switzerland.

†To whom correspondence should be addressed. Email: hafen@zool.unizh.ch

Fig. 1. Reduced kinase activity caused by an amino acid substitution in the $\mathrm{PH}$ domain of dAkt. (A) Effect of the G99S substitution in the $\mathrm{PH}$ domain on $\mathrm{dAkt}$ kinase activity from larval extracts (42). Activity from wild-type larvae was considered to be $100 \%$. Inset, dAkt matic activity appears to be the dephosphorylation of phosphoinositides at the D3 position. Because PTEN uses the second messenger PIP3 as a substrate, PTEN antagonizes the function of phosphatidylinositol-3 kinase (PI3K) $(7,8)$. Immortalized mouse embryonic fibroblasts or embryonic stem cells lacking PTEN function show an approximately twofold increase in PIP3 concentrations $(9,10)$. PIP3 interacts with a wide variety of $\mathrm{PH}$ domain-containing proteins, including the serine-threonine kinases Akt (also called $\mathrm{PKB}$ ) and phosphoinositide-dependent kinase 1 (PDK1), Btk family tyrosine kinases, guanine nucleotide exchange factors for the Rho and Arf families of small guanosine triphosphatases, and phospholipase $\mathrm{C} \gamma(11,12)$. The plethora of proteins that are potentially regulated by PIP3 provides widespread signaling potential for this lipid second messenger.

Genetic analyses in model organisms have implicated PTEN as a negative regulator of insulin receptor signaling. In the nematode Caenorhabditis elegans, PTEN antagonizes the activity of the PI3K AGE-1 in the regulation of metabolism, development, and life span (13-16). In the fruit fly Drosophila melanogaster, PTEN counteracts signaling downstream of the insulin receptor to control cellular growth (17-19). There are, however, focal adhesion kinase (5) and the adapte protein Shc (6), PTEN's predominant enzy-
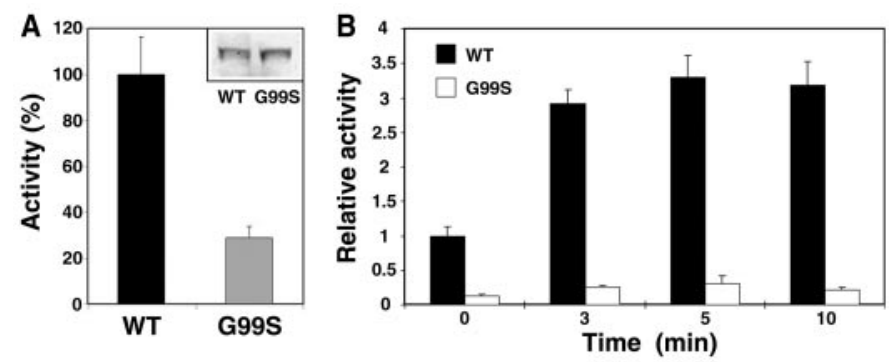

protein was detected in $40 \mu \mathrm{g}$ of larval extracts using the same antiserum. (B) Reduced insulin-induced activation of the G99S mutant dAkt. The dAkt constructs were expressed in HEK 293 cells (43). Transfected cells were starved for $\mathbf{2 4}$ hours before stimulation with insulin for the indicated time periods, and dAkt kinase activity was determined (44). The activity of wild-type dAkt from unstimulated cells was considered to be relative activity $=1$. 
additional phenotypes associated with mutations in PTEN that cannot easily be reconciled with an exclusive function of PTEN in insulin receptor signaling [for example, the burst vulva phenotype in C. elegans (13) and defects in the actin cytoskeleton in Drosophila (17)]. To better understand the consequences of loss of PTEN function, it would be useful to know which important downstream effectors react to increased PIP3 concentrations and whether PTEN has other physiological substrates in addition to PIP3.

The protein kinase Akt is an important component of insulin receptor signaling (20). Akt is recruited to the plasma membrane by virtue of the interaction of its $\mathrm{NH}_{2}$-terminally located PH domain with PIP3. At the membrane, subsequent phosphorylation events by
PDK1 and an unidentified kinase lead to the full activation of Akt (21-23). In PTENdeficient mouse embryonic fibroblasts and embryonic stem cells, Akt is phosphorylated and activated $(9,10)$. The phenotypes associated with Akt mutations in both $C$. elegans and Drosophila are consistent with its role in signal transduction downstream of the insulin receptor (24-27).

We monitored three properties of Drosophila Akt (dAkt) separately: kinase activity, abundance of the protein, and membrane localization. We relied entirely on mutations in the endogenous gene encoding dAkt to avoid potential side effects caused by overexpression of mutant proteins. $d A k t^{l}$ encodes a catalytically inactive protein, dAktF327I (25). The viable $d A k t^{4226}$ allele

Fig. 2. Reduced membrane localization of the G99S mutation of dAkt. HEK 293 cells plated on coverslips were transfected with epitope-tagged wildtype (A to C), G99S (D to F), and F327I (G to J) dAkt and deprived of serum for 16 hours before stimulation with insulin $(B, E, H)$ or pervanadate $(C, F, J)$ for 5 min. Fixed and permeabilized cells were incubated first with the monoclonal antibody 12CA5 to the HA epitope and then with fluorescein isothiocyanate-conjugated secondary antibody. An analysis by confocal microscopy revealed the subcellular localization of the dAkt variants.
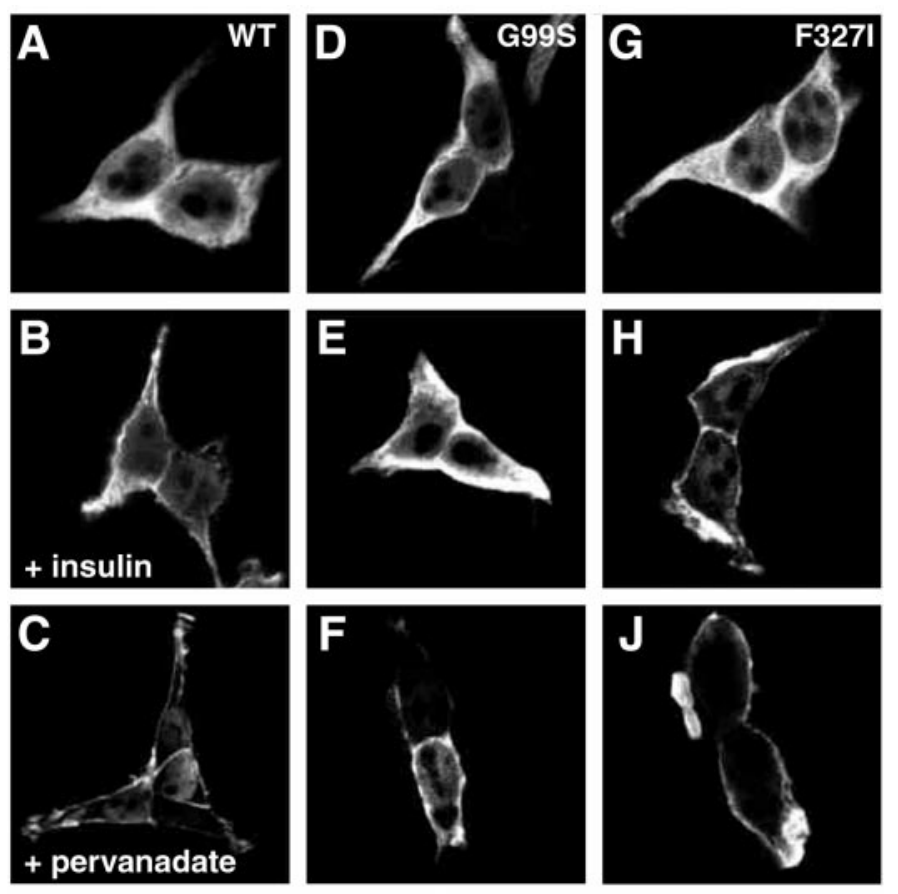

contains a P-element insertion upstream of the $d A k t$ gene and therefore results in the reduced expression of wild-type dAkt protein $(19,28)$. Finally, we characterized the viable hypomorphic mutation $d A k t^{3}$ (29) that selectively impairs the membrane recruitment of dAkt in response to increased concentrations of PIP3. Sequencing of genomic DNA extracted from $d A k t^{3}$ homozygous flies revealed a single nucleotide exchange resulting in the substitution of a serine residue for a nonconserved glycine at the end of the sixth $\beta$ sheet of the $\mathrm{PH}$ domain. To address the mechanism by which this Gly ${ }^{99} \rightarrow$ Ser $^{99}$ (G99S) mutation in the PH domain affects dAkt, we compared the amount of dAkt protein and activity in wild-type and $d A k t^{3}$ mutant larvae. Whereas no apparent difference in expression of the protein was observed (Fig. 1A, inset), dAkt activity from the mutant larvae represented only $30 \%$ of that in wild-type larval extracts (Fig. 1A). We also expressed epitope-tagged forms of wild-type dAkt, catalytically inactive dAktF327I, and PH domain mutant dAktG99S in insulin-responsive human embryonic kidney (HEK) 293 cells. All three proteins were expressed in similar amounts (30). dAktG99S activity from insulin-stimulated cells was reduced by about $90 \%$ as compared to that of the wild-type kinase (Fig. 1B). All forms of dAkt proteins were detected in the cytosol of unstimulated cells (Fig. 2, A, D, and G). Stimulation of the cells with insulin for 5 min resulted in association of the wild-type and the catalytically inactive enzymes with the plasma membrane, but failed to recruit the dAktG99S mutant protein (Fig. 2, B, E, and $\mathrm{H}$ ). In contrast, treatment of HEK 293 cells with the protein-tyrosine phosphatase inhibitor pervanadate, a potent activator of Akt (31), led to membrane recruitment of all dAkt proteins (Fig. 2, C, F, and J).
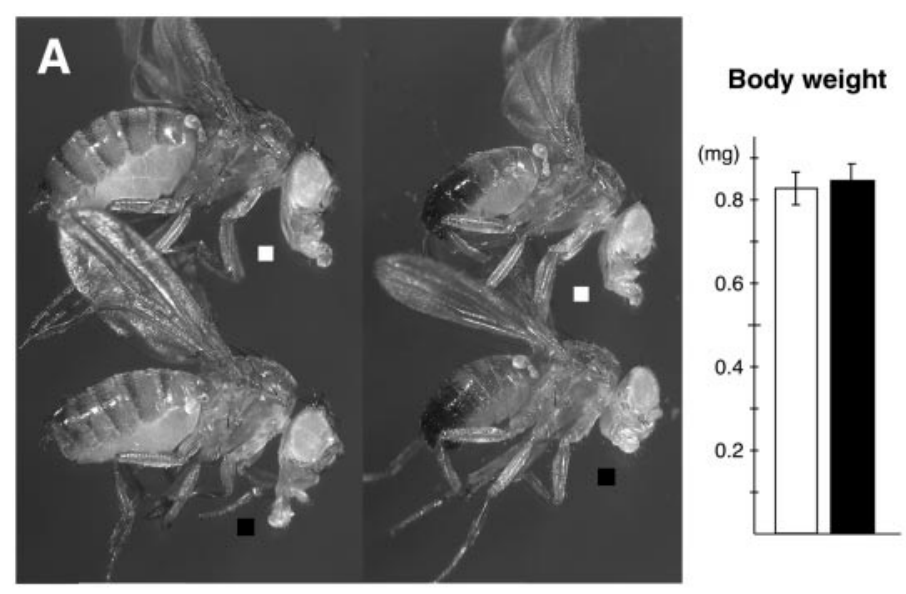

Fig. 3. Restored viability of flies lacking dPTEN function by the PH domain mutation in dAkt. (A) Morphology and weight of DPTEN mutant flies rescued by $d A k t^{3} / d A k t^{1}$. The left panel shows female flies, the middle

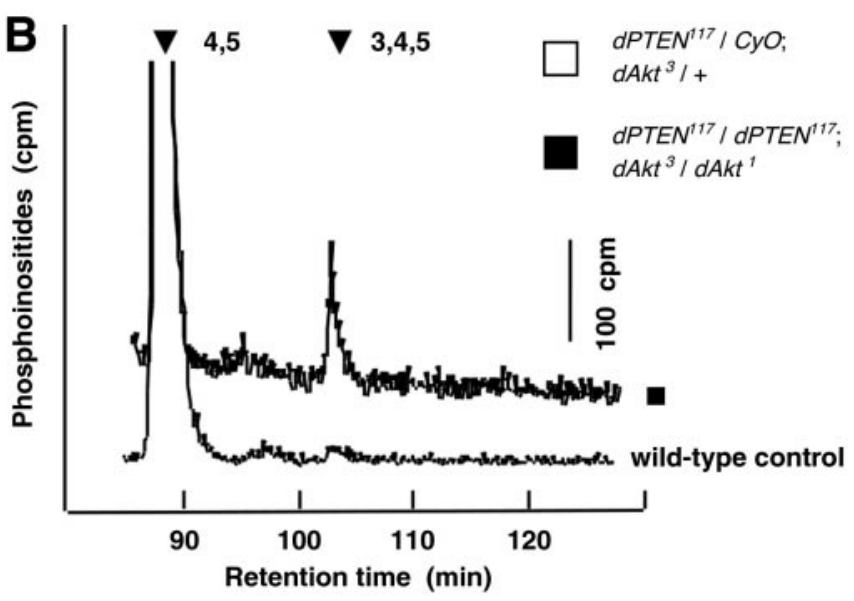

panel shows male flies, and the right panel shows the weight of adult male flies. (B) PIP3 concentrations in flies devoid of DPTEN function rescued by the $d A k t^{3}$ mutation. 

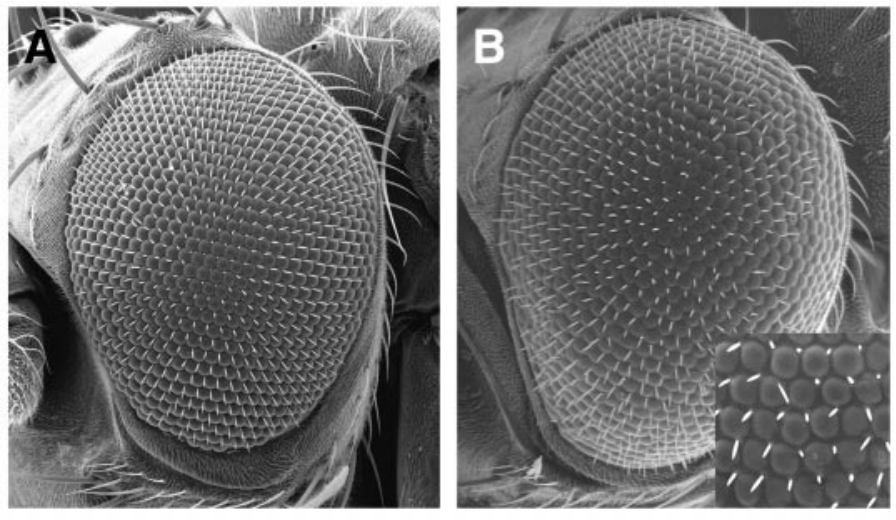

Fig. 4. Growth in the developing Drosophila eye promoted by activated dAkt. (A to D) Scanning electron micrographs of compound eyes of female flies; the anterior is to the left. (A) Wild type. (B) Overexpressing
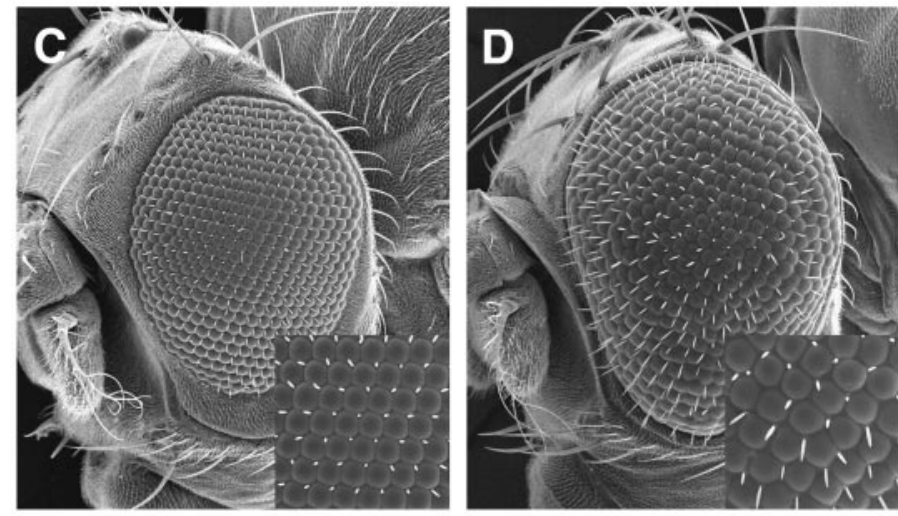

a membrane-tethered version of dAkt (GMR-Gal4 UAS-myr-dAkt). (C) chico mutant. (D) GMR-Gal4 UAS-myr-dAkt in a chico mutant background (45).
Consistently, pervanadate treatment stimulated dAktG99S activity to $80 \%$ of the wild-type level. However, pervanadate-induced activation of the mutant protein occurred more slowly than did that of the wild-type kinase (30). Taken together, these data indicate that the G99S substitution reduces the association of dAkt with the plasma membrane, probably by affecting the affinity of its PH domain for PIP3. Thus, $d A k t^{3}$ enabled us to study the consequences of impaired recruitment of dAkt to the plasma membrane.

We combined the $d A k t$ alleles with null mutations in $d P T E N$ (32). Animals lacking dPTEN function die during larval stages. A reduction in $d A k t$ expression using the viable $d A k t^{4226}$ allele did not rescue the lethality associated with $A P T E N$. Similarly, animals doubly mutant for $d P T E N$ and $d A k t^{l}$ did not survive. Thus, either dAkt activation is not the sole reason for the lethality caused by loss of PTEN, or dAkt function is not dispensable in the absence of dPTEN. The latter hypothesis is strongly supported by results obtained with the $d A k t$ allele that selectively impairs the membrane recruitment of dAkt. Flies devoid of functional dPTEN were rescued to viability by any $d A k t$ allelic combination that included $d A k t^{3}$ (Fig. 3A) (33). The rescued flies did not display morphological defects that would be expected in light of the phenotypes ascribed to clones of $d P T E N$ mutant cells (17). Tangential sections through compound eyes revealed essentially normal ommatidial and rhabdomeric structures, and the wings of the rescued flies showed no abnormalities in the venation, such as missing crossveins (34). We determined the PIP3/ PIP2 ratio by metabolic labeling of phospholipids from larvae (35). PIP3 concentrations were increased in the dPTEN dAkt doubly mutant larvae as compared to those of wildtype larvae (Fig. 3B), excluding the possibility that PIP3 concentrations remain within physiological limits by a feedback regulation mechanism involving dAkt. This suggests that the potential activation of a number of $\mathrm{PH}$ domain-containing proteins other than dAkt does not interfere with viability.

Our results indicate that the activation of dAkt is the only crucial outcome of the loss of dPTEN function. Activation of dAkt should therefore mimic the dPTEN loss-offunction phenotype. We expressed a constitutively activated membrane-anchored dAkt during eye development (36). The resulting eyes were increased in size due to enlarged ommatidia (Fig. 4B), a phenotype similar to that seen in clones of $A P T E N$ mutant cells (17-19). This overgrowth phenotype is independent of upstream signals, because it was still evident in a chico or Dp110/PI3K mutant background (Fig. 4D) (34).

We conclude that flies devoid of the tumor suppressor dPTEN can live with abnormally high concentrations of PIP3 if only the affinity of dAkt for PIP3 is decreased. Thus, the $\mathrm{PH}$ domain-mediated translocation of dAkt to the membrane and its subsequent activation is the only lethal event triggered by increased PIP3 concentrations. Because the $\mathrm{PH}$ domain of Akt interacts with the substrate of PTEN's lipid phosphatase activity, we also conclude that PTEN does not exert any essential function other than the dephosphorylation of PIP3.

\section{References and Notes}

1. L. Simpson, R. Parsons, Exp. Cell Res. 264, 29 (2001).

2. J. Li et al., Science 275, 1943 (1997).

3. P. A. Steck et al., Nature Genet. 15, 356 (1997).

4. D. M. Li, H. Sun, Cancer Res. 57, 2124 (1997).

5. M. Tamura et al., Science 280, 1614 (1998).

6. J. Gu et al., J. Cell Biol. 146, 389 (1999).

7. T. Maehama, J. E. Dixon, J. Biol. Chem. 273, 13375 (1998)

8.

9. V. Stambolic et al., Cell 95, 29 (1998).

10. H. Sun et al., Proc. Natl. Acad. Sci. U.S.A. 96, 6199 (1999).

11. B. Vanhaesebroeck et al., Annu. Rev. Biochem. 70, 535 (2001).

12. S. J. Leevers, B. Vanhaesebroeck, M. D. Waterfield, Curr. Opin. Cell Biol. 11, 219 (1999).

13. S. Ogg, G. Ruvkun, Mol. Cell 2, 887 (1998).
14. E. B. Gil, E. Malone Link, L. X. Liu, C. D. Johnson, J. A Lees, Proc. Natl. Acad. Sci. U.S.A. 96, 2925 (1999).

15. V. T. Mihaylova, C. Z. Borland, L. Manjarrez, M. J. Stern, H. Sun, Proc. Natl. Acad. Sci. U.S.A. 96, 7427 (1999).

16. J. P. Rouault et al., Curr. Biol. 9, 329 (1999)

17. D. C. I. Goberdhan, N. Paricio, E. C. Goodman, M. Mlodzik, C. Wilson, Genes Dev. 13, 3244 (1999).

18. H. Huang et al., Development 126, 5365 (1999).

19. X. Gao, T. P. Neufeld, D. Pan, Dev. Biol. 221, 404 (2000).

20. M. A. Lawlor, D. R. Alessi, J. Cell Sci. 114, 2903 (2001).

21. J. Downward, Curr. Opin. Cell Biol. 10, 262 (1998).

22. P. J. Coffer, J. Jin, J. R. Woodgett, Biochem. J. 335, 1 (1998).

23. T. O. Chan, S. E. Rittenhouse, P. N. Tsichlis, Annu. Rev. Biochem. 68, 965 (1999)

24. S. Paradis, G. Ruvkun, Genes Dev. 12, 2488 (1998).

25. B. E. Staveley et al., Curr. Biol. 8, 599 (1998).

26. J. Verdu, M. A. Buratovich, E. L. Wilder, M. J. Birnbaum, Nature Cell Biol. 1, 500 (1999).

27. S. E. Scanga et al., Oncogene 19, 3971 (2000).

28. A. C. Spradling et al., Genetics 153, 135 (1999).

29. The $d A k t^{3}$ allele was found in a collection of homozygous viable mutations from $\mathrm{Ch}$. Zuker and $\mathrm{E}$. Koundakjian (University of California, San Diego) because it yields flies of severely reduced body size.

30. M. Andjelkovic, B. A. Hemmings, unpublished results.

31. M. Andjelkovic et al., Proc. Natl. Acad. Sci. U.S.A. 93, 5699 (1996).

32. The $A P T E N$ alleles used are $\operatorname{APTEN}^{d j 189}$ (19) and DPTEN $^{117}$ (37). Both are presumably null alleles. We also tested them over a deficiency lacking the $D P T E N$ locus, $D f(2 L) 170 B$. Because this deficiency also lacks chico, an upstream component of insulin receptor signaling, we reintroduced chico by means of a genomic rescue construct. In all cases, we obtained similar results.

33. Whereas the strongest allelic combination $d A k t^{1} /$ $d A k t^{3}$ completely rescued the $d P T E N$ mutant flies to wild-type size, the combinations $d A k t^{3} / d A k t^{3}$ and $d A k t^{3} / d A k t^{4226}$ could rescue the lethality associated with loss of PTEN function, but the resulting flies were slightly enlarged. Furthermore, we observed a variability of the phenotypes in all combinations. Consistently, flies that emerged earlier showed a tendency to be of increased size, whereas some retarded flies were of smaller size.

34. H. Stocker, E. Hafen, unpublished results.

35. Nonwandering third instar larvae were phosphate starved in phosphate-free Schneider S2 medium and then labeled with $2 \mathrm{mCi}$ per sample of inorganic ${ }^{32} \mathrm{P}$ (50 $\mathrm{mCi} / \mathrm{ml})$. PIP3 and PIP2 levels were determined according to (38).

36. To anchor dAkt to the plasma membrane, the myristoylation-palmitoylation motif from the Lck tyrosine kinase was fused to the $\mathrm{NH}_{2}$-terminus of hemagglutinin (HA) epitope-tagged dAkt, as previously described for mammalian Akt (39). Analysis of the subcellular localization by immunofluorescence using the epitope antibody to HA confirmed the constitutive membrane localization of myr-dAkt. 
37. S. Oldham, E. Hafen, unpublished results.

38. A. Arcaro, M. P. Wymann, Biochem. J. 296, 297 (1993).

39. M. Andjelkovic et al., J. Biol. Chem. 272, 31515 (1997).

40. M. Andjelkovic et al., J. Biol. Chem. 270, 4066 (1995).

41. Single-letter abbreviations for the amino acid residues are as follows: A, Ala; E, Glu; F, phe; G, Gly; I, Ile; P, Pro; R, Arg; S, Ser; and T, Thr.

42. dAkt was immunoprecipitated from wild-type and $d A k t^{3}$ larvae with a rabbit polyclonal antibody raised against recombinant dAkt-66 (40). In vitro kinase assays were performed, as described, using the peptide GRPRTSSAEG (41) as a substrate (39).

43. HEK 293 cells were transfected by a modified calcium phosphate method, as described (39). The transfec- tion mixture was removed after a 16-hour incubation, and cells were serum-starved for 24 hours before stimulation with 0.5 to $1 \mu \mathrm{M}$ insulin (Boehringer Mannheim).

44. The HA epitope-tagged dAkt proteins were immunoprecipitated from $100 \mu \mathrm{g}$ of cell-free extracts using the monoclonal antibody 12CA5 coupled to protein A-Sepharose. In vitro kinase assays were performed, as described (39).

45. The size difference of the eyes in Fig. $4, B$ and $D$, is entirely due to varying numbers of ommatidia. The size of the ommatidia, however, is comparable (insets) and massively larger than in chico mutants (inset in 4C). The failure of myr-dAkt to compensate for the reduced number of ommatidia in chico mu- tants is consistent with the late onset of expression driven by GMR-Gal4.

46. We thank Ch. Zuker and E. Koundakjian for the $d A k t^{3}$ allele; T. Radimerski for the S2 labeling medium; T. Gutjahr, Ch. Hugentobler, R. Bopp, P. Zipperlen, P. Cron, P. Müller, and H. Angliker for technical support; K. Basler and P. Gallant for critical reading of the manuscript and valuable suggestions; and D. Pan and S. Leevers for providing fly stocks. Supported by grants from the Schweizerische Krebsliga (B.A.H. and M.P.W.) and the Swiss National Science Foundation (E.H.). 